\title{
Adverse drug reactions and nursing interventions in the treatment of non-Hodgkin's lymphoma by Aidi injection
}

\author{
Lian Yan, Wenjuan Li, Shanshan He, Aifeng Meng, Jianhong Liu, Jianmei Zhou \\ Department of Nurse Station, Jiangsu Cancer Hospital Jiangsu Institute of Cancer Prevention and Treatment, Area 211, Affiliated Tumor Hospital \\ of Nanjing Medical University, Nanjing, China \\ Contributions: (I) Conception and design: L Yan, W Li; (II) Administrative support: A Meng; (III) Provision of study materials or patients: S He; (IV) \\ Collection and assembly of data: J Zhou; (V) Data analysis and interpretation: J Liu; (VI) Manuscript writing: All authors; (VII) Final approval of \\ manuscript: All authors. \\ Correspondence to: Jianmei Zhou. Department of nurse station, Jiangsu Cancer Hospital Jiangsu Institute of Cancer Prevention and Treatment; Area \\ 211, Affiliated Tumor Hospital of Nanjing Medical University, Nanjing 210009, China. Email: 1172806794@qq.com.
}

Background: To explore adverse drug reactions (ADRs) and the effects of nursing interventions after Aidi injection for the treatment of non-Hodgkin's lymphoma (NHL).

Methods: A total of 104 NHL patients treated in our hospital from March 2019 to March 2020 were selected. All patients underwent conventional chemotherapy, with a concomitant Aidi injection administered at different doses (40, 60, 80, and $100 \mathrm{~mL}$ ). ADRs were analyzed for all patients, and the clinical outcomes of ADRs were recorded after specific nursing interventions were performed.

Results: A total of 17 NHL patients had ADRs, with a total incidence rate of $15.89 \%$ (total of 30 ADRs). In terms of different types of ADRs, inappetence accounted for the largest proportion, followed by skin pruritus and fever, phlebitis, nausea and vomiting, and chest distress and/or palpitation, the last of which shared the same proportion as chills and/or low fever and urticaria. The incidence rate of ADRs (total of 18 ADRs, $60.00 \%$ ) was higher in NHL patients who were given $100 \mathrm{~mL}$ of Aidi injection. In terms of age and gender distribution of ADRs, there were 10 males (55.56\%) and 7 females (44.44\%), and ADRs were more common in patients aged 46-60 years old (total of 6 ADRs, 35.29\%). Aidi injection mainly induced mild ADRs (total of 22 ADRs, 73.33\%), and the resulting ADRs mostly occurred for <2 hours (total of 19 ADRs, $60.00 \%)$. After the specific nursing interventions were performed, no deaths due to ADRs occurred, and 12 (64.71\%) cases were cured, 5 (29.41\%) cases improved, and 1 (5.88\%) case had no progression.

Conclusions: Particular attention should be paid to ADRs in the treatment of NHL patients with Aidi injection. After ADRs occur, specific nursing interventions can aid in recovery and lead to improvements in prognosis.

Keywords: Aidi injection; non-Hodgkin's lymphoma (NHL); adverse drug reactions (ADRs); nursing intervention

Submitted Sep 10, 2020. Accepted for publication Nov 17, 2020.

doi: 10.21037/apm-20-1990

View this article at: http://dx.doi.org/10.21037/apm-20-1990

\section{Introduction}

Non-Hodgkin's lymphoma (NHL) is a malignant tumor originating from the lymph nodes or lymph tissues, manifesting as painless and progressive lymph node enlargement or local mass, and accounts for approximately $90 \%$ of all malignant lymphomas (1). NHL is prone to diffusion in the early stage, with systemic and diverse symptoms. Currently, the clinical treatment of NHL is mainly chemotherapy, and vinorelbine, prednisone, and cyclophosphamide are the major therapeutic drugs (2-4). In recent years, due to an improvement in the status of traditional Chinese medicines, some Chinese herbal preparations with antitumor effects have also been applied 
in the clinical treatment of NHL.

As a Chinese herbal preparation, Aidi injection can clear away heat and remove toxicity, counteract toxins and remove sores, and eliminate blood stasis and dispel stagnation, making it useful for the treatment of malignant tumors (5). Aidi injection is mainly composed of cantharides, Panax ginseng, Acanthopanax senticosus, and Astragalus membranaceus. Cantharides can dissipate blood stasis and swollen masses. $P$. ginseng, often referred to as "king of herbs", is understood to reinforce vital energy, induce resistance to fatigue, and improve immunity (6). A. senticosus can regulate qi-blood function, accelerate metabolism, promote blood circulation, and enhance the body's resistance and endurance (7). A. membranaceus is able to invigorate qi for consolidating superficies, promote urination and tissue regeneration, stimulate the proliferation of bone marrow cells, and regulate the immunocompetence and phagocytic function of phagocytes (8). At present, the antitumor effects of $A$. membranaceus, A. senticosus, and cantharides are welldocumented (9-11). Additionally, lymphoma has been effectively treated by Aidi injection (12). Despite demonstrating efficacy, there are also many reports regarding adverse drug reactions (ADRs) caused by Chinese herbal preparations in recent years (13). However, ADRs induced by the Chinese herbal preparation Aidi injection remain rare. It is therefore of great significance to analyze Aidi injection-induced ADRs, enhance the awareness of drug-induced ADRs, and take necessary preventive measures to reduce ADRs as much as possible. Towards this end, we analyzed Aidi injection-induced ADRs in this study, and a specific nursing regimen was utilized for ADR intervention, aiming to provide a theoretical basis for the promotion and application of Aidi injection in NHL treatment.

We present the following article in accordance with the TREND reporting checklist (available at http://dx.doi. org/10.21037/apm-20-1990).

\section{Methods}

\section{Baseline clinical data}

A total of 104 NHL patients treated in our hospital from March 2019 to March 2020 were selected, including 54 males and 50 females with an average age of $46.86 \pm$ 9.15 years. The inclusion criteria were as follows: (I) patients diagnosed with NHL according to the WHO criteria (14);
(II) patients with a Karnofsky performance scale score $\geq 60$ points and an expected survival time $\geq 6$ months; (III) patients without other organic lesions; (IV) patients who were Aidi injection-naïve. The exclusion criteria were as follows: (I) NHL patients who had previously had Aidi injections; (II) patients with mental illnesses or cognitive impairment; (III) patients who did not agree to participate or could not participate in the study for other reasons.

All procedures performed in this study involving human participants were in accordance with the Declaration of Helsinki (as revised in 2013). The study was approved by institutional ethics board of Jiangsu Cancer Hospital (NO.2019-028) and informed consent was taken from all the patients.

\section{Study methods}

\section{Administration methods}

All NHL patients underwent conventional chemotherapy as follows: on the 1 st day of treatment, patients were intravenously infused with cyclophosphamide, pirarubicin, and vinorelbine at doses of 750,40 , and $30 \mathrm{mg} / \mathrm{m}^{2}$, respectively. Within the first week of treatment, prednisone was taken orally at $40 \mathrm{mg} / \mathrm{m}^{2}$ once a day. Based on conventional chemotherapy, Aidi injection was intravenously infused at a single dose of $40,60,80$, or $100 \mathrm{~mL}$ for 26 patients each once a day, and a $5 \%$ glucose injection was used as the solvent. One course of treatment lasted for 14 days, and the next course commenced after an interval of 7 days.

\section{Clinical nursing intervention}

Patients underwent a clinical nursing intervention, which was performed as follows:

(I) Nursing for gastrointestinal reactions: high-calorie, high-protein and high-fiber semi-liquid food was provided at an appropriate time according to the actual condition of the patient. The time interval of consumption was observed, and the patients did not rest in bed immediately after eating. For cases of severe gastrointestinal reactions, drugs were withdrawn immediately, and $10 \mathrm{mg}$ of metoclopramide was intramuscularly injected.

(II) Nursing for skin and appendage reactions: in the case of phlebitis, the drug was injected into thick, straight, and flexible veins as far as possible, generally from the distal to the proximal end and 
from the dorsal to the medial side alternately on the left and right hands. The drug was not administered through the lower limb veins, and if conditions permitted, the indwelling needle or central venous catheter was chosen, with the port retained. During the infusion, the infusion rate was 15 drops/minute at the beginning, then it was controlled at 50 drops/ minute if there were no ADRs after 30 minutes, aiming to reduce local irritation to blood vessels. Patients with skin pruritus and fever or urticaria contacted doctors promptly and received timely and effective treatment. In severe cases, the drug was withdrawn immediately, and the corresponding medication was taken as directed by the doctor.

(III) If chest distress, palpitations, low fever, and chills occurred, the drug was withdrawn immediately. Patients with severe chest distress and palpitations contacted doctors promptly to undergo symptomatic treatments such as oxygen inhalation as directed by the doctor.

(IV) Mental nursing: nursing staff actively introduced and imparted knowledge about NHL and the importance of Aidi injection, and explained its ADRs in detail, aiming to improve patient awareness of the disease and avoid panic.

\section{Observation indices}

The characteristics of Aidi injection-induced ADRs in NHL patients were recorded, including the time of occurrence, clinical manifestations, symptom grade, and clinical outcome. ADRs were graded as follows: (15) (I) mildtransient allergic reactions that recovered spontaneously without any medical intervention, (II) moderate-transient ADRs that required medical intervention but were not life threatening and disappeared gradually, (III) severe-longlasting ADRs that involved multiple organ systems, could not be fully recovered after medical intervention, and were life-threatening, (IV) death.

\section{Statistical analysis}

In this retrospective study, patients' gender, age, clinical characteristics, and outcomes of ADRs were collected, and Microsoft Office Excel was used for data sorting, classification, and statistical analysis.

\section{Results}

\section{ADRs after administration of different doses of Aidi injection}

A total of 17 NHL patients had ADRs, and the total incidence rate was $15.89 \%$ (total of 30 ADRs). In terms of different types of ADRs, inappetence accounted for the largest proportion, followed by skin pruritus and fever, phlebitis, nausea and vomiting, and chest distress and/or palpitation, the last of which shared the same proportion as chills and/or low fever and urticaria. No ADRs occurred in NHL patients in the $40 \mathrm{~mL}$ group, and there was a total of $3(10.00 \%)$ ADRs in the $60 \mathrm{~mL}$ group, $9(30.00 \%)$ ADRs in the $80 \mathrm{~mL}$ group, and $18(60.00 \%)$ ADRs in the $100 \mathrm{~mL}$ group (Table 1).

\section{Age and gender distribution of ADRs}

In terms of the gender distribution of patients with ADRs, there were 10 males (55.56\%) and 7 females (44.44\%). Also, ADRs were more common in patients aged 46-60 years old (total of 6 ADRs, 35.29\%) (Table 2).

\section{Grading and duration of ADRs}

Aidi injection mainly induced mild ADRs (total of 22 ADRs, $73.33 \%$ ), and the resulting ADRs mostly occurred for $<2$ hours (total of 19 ADRs, $63.33 \%$ ) (Table 3).

\section{Clinical outcomes of ADRs}

After the specific nursing intervention was performed, no deaths due to ADRs occurred. Furthermore, 12 (64.71\%) cases were cured, 5 (29.41\%) cases improved, and 1 (5.88\%) case had no progression.

\section{Discussion}

Aidi injection is a scientifically formulated novel biphasic anti-cancer drug administered via intravenous infusion which can exert immunoregulatory effects and may aid in cancer resistance. Liu et al. reported that the major pharmacological effects of Aidi injection included radiosensitization, induction of cancer cell apoptosis, and inhibition of cancer cell proliferation (16). Aidi injection has 
Table 1 Organs and systems impacted by adverse drug reactions (ADRs) following Aidi injection at different doses

\begin{tabular}{|c|c|c|c|c|c|}
\hline Aidi injection dose & $40 \mathrm{~mL}$ & $60 \mathrm{~mL}$ & $80 \mathrm{~mL}$ & $100 \mathrm{~mL}$ & Proportion \\
\hline \multicolumn{6}{|l|}{ Digestive system } \\
\hline Inappetence & 0 & 1 & 5 & 7 & $13(43.33 \%)$ \\
\hline Nausea and vomiting & 0 & 0 & 1 & 2 & $3(10.00 \%)$ \\
\hline \multicolumn{6}{|l|}{ Skin and appendages } \\
\hline Pruritus and fever & 0 & 1 & 2 & 3 & $6(20.00 \%)$ \\
\hline Urticaria & 0 & 0 & 0 & 1 & $1(3.33 \%)$ \\
\hline Phlebitis & 0 & 1 & 1 & 2 & $4(13.33 \%)$ \\
\hline \multicolumn{6}{|l|}{ Other } \\
\hline Chill and/or low fever & 0 & 0 & 0 & 1 & \\
\hline Chest distress and/or palpitation & 0 & 0 & 0 & 1 & $1(3.33 \%)$ \\
\hline Total & 0 & 3 (10.00\%) & $9(30.00 \%)$ & $18(60.00 \%)$ & $30(100.00 \%)$ \\
\hline
\end{tabular}

Table 2 Age and gender distribution of adverse drug reactions (ADRs) following Aidi injection

\begin{tabular}{lccc}
\hline Age (year) & Male & Female & Total \\
\hline $18-30$ & 2 & 1 & $3(17.65 \%)$ \\
$31-45$ & 3 & 2 & $5(29.41 \%)$ \\
$46-60$ & 3 & 3 & $6(35.29 \%)$ \\
$>60$ & 2 & 1 & $3(17.65 \%)$ \\
Total & $10(58.82 \%)$ & $7(41.18 \%)$ & $17(100.00 \%)$ \\
\hline
\end{tabular}

Table 3 Grading and duration of adverse drug reactions (ADRs)

\begin{tabular}{lccc}
\hline Duration & Mild & Moderate & Total \\
\hline$<2$ hours & 14 & 5 & $19(63.33 \%)$ \\
$2-24$ hours & 5 & 2 & $7(23.33 \%)$ \\
$24-48$ hours & 2 & 1 & $3(10.00 \%)$ \\
$48-72$ hours & 1 & 0 & $1(3.33 \%)$ \\
Total & $22(73.33 \%)$ & $8(26.67 \%)$ & $30(100.00 \%)$ \\
\hline
\end{tabular}

demonstrated efficacy for a variety of cancers, such as lung cancer (14). Recently, its therapeutic effects on lymphoma have also been reported (12).

ADRs refer to the harmful reactions that can occur under normal usage and dosages which are unrelated to drug medicinal effects. ADRs are closely related to the prognosis of disease, and therefore, attention needs to be paid to the monitoring of ADRs. However, there is a notion that "pure Chinese herbal preparations have no toxicity or side effects at all" among Chinese people, and no emphasis has been placed on investigating the ADRs of Chinese herbal preparations for a long time. With the increasing consumption of herbal products intended to promote better health, it is therefore extremely important to assure the safety and quality of herbal preparations (15). The increasing application of Aidi injection for the treatment of cancer has meant that the resulting ADRs have been recently reported. Xiao et al. reported that the adverse reactions of Faxianaidi injection were mainly hematogenous toxicity, gastrointestinal toxicity, liver and kidney injury, neurotoxicity, and hair loss (13).

Similar to the above study, the total incidence rate of ADRs in this study was $15.89 \%$ (total of 30 ADRs) in the treatment of NHL patients with Aidi injection. The main $\mathrm{ADR}$ was inappetence, followed by skin pruritus and fever, indicating that Aidi injection exerted significant effects on the digestive system. In addition, this study also found that Aidi injection impacted the skin and appendages. The normal dose of Aidi injection generally ranges from 50$100 \mathrm{~mL}$. In this study, ADRs most frequently occurred in NHL patients in the $100 \mathrm{~mL}$ dose group. The above findings suggest that when the dose of Aidi injection is higher, ADRs occur more frequently due to increased toxicity and side effects. There was little difference in the gender distribution of NHL patients with ADRs, and they 
were more common in patients aged 46-60 years old. In terms of ADR duration and grade, Aidi injection mainly induced mild ADRs (total of 22 ADRs, $73.33 \%$ ), and the resulting ADRs mostly occurred for $<2$ hours (total of 19 ADRs, 63.33\%). After the specific nursing intervention was performed, no deaths due to ADRs occurred, and 12 (64.71\%) cases were cured, 5 (29.41\%) cases improved, and $1(5.88 \%)$ case had no progression. Hence, Aidi injection induced short-lasting and milder ADRs in the treatment of NHL, and prognosis improved after a specific nursing intervention was applied.

In conclusion, particular attention should be paid to ADRs after Aidi injection for the treatment of NHL. After ADRs occur, specific nursing interventions can aid in recovery and lead to improvements in prognosis.

\section{Acknowledgments}

Funding: None.

\section{Footnote}

Reporting Checklist: The authors have completed the TREND reporting checklist. Available at http://dx.doi. org/10.21037/apm-20-1990

Data Sharing Statement: Available at http://dx.doi. org/10.21037/apm-20-1990

Conflicts of Interest: All authors have completed the ICMJE uniform disclosure form (available at http://dx.doi. org/10.21037/apm-20-1990). The authors have no conflicts of interest to declare.

Ethical Statement: The authors are accountable for all aspects of the work in ensuring that questions related to the accuracy or integrity of any part of the work are appropriately investigated and resolved. All procedures performed in this study involving human participants were in accordance with the Declaration of Helsinki (as revised in 2013). The study was approved by institutional ethics board of Jiangsu Cancer Hospital (NO.2019-028) and informed consent was taken from all the patients.

Open Access Statement: This is an Open Access article distributed in accordance with the Creative Commons Attribution-NonCommercial-NoDerivs 4.0 International License (CC BY-NC-ND 4.0), which permits the non- commercial replication and distribution of the article with the strict proviso that no changes or edits are made and the original work is properly cited (including links to both the formal publication through the relevant DOI and the license). See: https://creativecommons.org/licenses/by-nc-nd/4.0/.

\section{References}

1. Hochberg J, Flower A, Brugieres L, et al. NHL in adolescents and young adults: A unique population. Pediatr Blood Cancer 2018;65:e27073.

2. Keating GM. Pixantrone: A Review in Relapsed or Refractory Aggressive Non-Hodgkin's Lymphoma. Drugs 2016;76:1579-86.

3. Barrenetxea Lekue C, Grasso Cicala S, Leppä S, et al. Pixantrone beyond monotherapy: a review. Ann Hematol 2019;98:2025-33.

4. Patel DA. Haploidentical Stem Cell Transplantation With Post-Transplantation Cyclophosphamide for Aggressive Lymphomas: How Far Have We Come and Where Are We Going? World J Oncol 2019;10:1-9.

5. Liu L, Liang J, Deng X. Effects of Aidi Injection with Western Medical Therapies on Quality of Life for Patients with Primary Liver Cancer: A Systematic Review and Meta-Analysis. Chin J Integr Med 2019;25:785-90.

6. Nguyen NH, Nguyen CT. Pharmacological effects of ginseng on infectious diseases. Inflammopharmacology 2019;27:871-83.

7. Li T, Ferns K, Yan ZQ, et al. Acanthopanax senticosus: Photochemistry and Anticancer Potential. Am J Chin Med 2016;44:1543-58.

8. Zhang J, Wu C, Gao L, et al. Astragaloside IV derived from Astragalus membranaceus: A research review on the pharmacological effects. Adv Pharmacol 2020;87:89-112.

9. Auyeung KK, Han QB, Ko JK. Astragalus membranaceus: A Review of its Protection Against Inflammation and Gastrointestinal Cancers. Am J Chin Med 2016;44:1-22.

10. Li T, Ferns K, Yan ZQ, et al. Acanthopanax senticosus: Photochemistry and Anticancer Potential. Am J Chin Med 2016;44:1543-58.

11. Puerto Galvis CE, Vargas Méndez LY, Kouznetsov VV. Cantharidin-based small molecules as potential therapeutic agents. Chem Biol Drug Des 2013;82:477-99.

12. Wang X, Jin W, Fang B, et al. Aidi injection combined with CHOP chemotherapy regimen in the treatment of malignant lymphoma: A meta-analysis based on randomized controlled trials. J Cancer Res Ther 2016;12:11-4. 
13. Xiao Z, Wang C, Zhou M, et al. Clinical efficacy and safety of Aidi injection plus paclitaxel-based chemotherapy for advanced non-small cell lung cancer: A meta-analysis of 31 randomized controlled trials following the PRISMA guidelines. J Ethnopharmacol 2019;228:110-22.

14. Xiao Z, Wang CQ, Zhou R, et al. Can Aidi injection improve overall survival in patients with non-small cell lung cancer? A systematic review and meta-analysis of 25 randomized controlled trials. Complement Ther Med 2018;37:50-60.

Cite this article as: Yan L, Li W, He S, Meng A, Liu J, Zhou J. Adverse drug reactions and nursing interventions in the treatment of non-Hodgkin's lymphoma by Aidi injection. Ann Palliat Med 2020;9(6):4038-4043. doi: 10.21037/apm-20-1990
15. Sun XF, Pei YT, Yin QW, et al. Application of Aidi injection in the bronchial artery infused neo-adjuvant chemotherapy for stage III A non-small cell lung cancer before surgical operation. Chin J Integr Med 2010;16:537-41.

16. Liu SH, Chuang WC, Lam W, et al. Safety surveillance of traditional Chinese medicine: current and future. Drug Saf 2015;38:117-28.

(English Language Editor: C. Betlazar-Maseh) 